\title{
Inelastic non-Newtonian flow over heterogeneously slippery surfaces
}

\author{
A. Sander Haase, Jeffery A. Wood, Lisette M. J. Sprakel, and Rob G. H. Lammertink* \\ Soft matter, Fluidics and Interfaces, MESA ${ }^{+}$Institute for Nanotechnology, University of Twente, \\ P.O. Box 217, 7500 AE Enschede, The Netherlands
}

(Received 15 September 2016; published 9 February 2017)

\begin{abstract}
In this study, we investigated inelastic non-Newtonian fluid flow over heterogeneously slippery surfaces. First, we simulated the flow of aqueous xanthan gum solutions over a bubble mattress, which is a superhydrophobic surface consisting of transversely positioned no-slip walls and no-shear gas bubbles. The results reveal that for shear-thinning fluids wall slip can be increased significantly, provided that the system is operated in the shear-thinning regime. For a $0.2 \mathrm{wt} \%$ xanthan gum solution with a power-law index of $n=0.4$, the numerical results indicate that wall slip can be enhanced 3.2 times when compared to a Newtonian liquid. This enhancement factor was also predicted from a theoretical analysis, which gave an expression for the maximum slip length that can be attained over flat, heterogeneously slippery surfaces. Although this equation was derived for a no-slip/no-shear unit length that is much larger than the typical size of the system, we found that it can also be used to predict the enhancement in the regime where the slip length is proportional to the size of the no-shear region or the bubble width. The results could be coupled to the hydrodynamic development or entrance length of the system, as maximum wall slip is only reached when the fluid flow can fully adapt to the no-slip and no-shear conditions at the wall.
\end{abstract}

DOI: 10.1103/PhysRevE.95.023105

\section{INTRODUCTION}

To enhance transport phenomena in microfluidic and nanofluidic systems, surfaces can be made slippery. The slipperiness of the surface not only leads to lower friction at the wall, and therefore higher flow rates for a given driving force, but also increases convection near the wall, improving transport of, for example, heat or mass. Navier's slip condition [1] is commonly used to quantify the amount of wall slip. This condition states that the slip velocity $u_{b}$ times a certain friction factor $k$ equals the shear force $\mu \partial_{n} u$ at the wall, i.e.,

$$
k u_{b}=\mu \frac{\partial u}{\partial n} .
$$

The ratio $\mu / k=b$ is referred to as the slip length and quantifies the amount of wall slip. For smooth surfaces displaying intrinsic wall slip - the fluid molecules adjacent to the substrate have a finite velocity relative to the wall-typical slip lengths are of the order of tens of nanometers [2]. Much larger slip lengths are observed for superhydrophobic surfaces, of the order of micrometers $[3,4]$, although this concerns effective wall slip. For these superhydrophobic surfaces, which contain (sub)microscale wall structures in which usually a gas is entrapped, the liquid is flowing over an array of no-slip regions and (nearly) shear-free gas bubbles. The effective slip length therefore represents the slip length that would be observed at scales much larger than those of the surface structures.

The fact that superhydrophobic surfaces can be used to generate slip lengths on the scale of a typical microfluidic system has led to many theoretical, numerical, and experimental

\footnotetext{
*r.g.h.lammertink@utwente.nl
}

Published by the American Physical Society under the terms of the Creative Commons Attribution 4.0 International license. Further distribution of this work must maintain attribution to the author(s) and the published article's title, journal citation, and DOI. studies in which the relationship between surface geometry and wall slip has been investigated [3-6]. These studies, however, mainly concern slip flow of Newtonian liquids over superhydrophobic surfaces.

The existence of wall slip in systems without superhydrophobic or patterned surfaces has been studied for a much longer time for a wide range of complex fluids, such as polymer melts, (bio)polymers solutions, suspensions, and gels [7-11]. The slip mechanisms in such systems can vary. Three possible apparent slip mechanisms are commonly considered in the literature, which all differ from true or intrinsic wall slip [5]. The first mechanism is the formation of a low-viscosity slip or depletion layer near the wall [9]. This layer, formed due to migration of the polymer chains toward the bulk of the flow, then acts as a lubrication layer over which the more viscous polymeric solution flows. The thickness of this layer is typically of the order of the size of the polymer coil, i.e., $\mathscr{O}(\mathrm{nm})$. Second, slippage can be the result of breaking the adhesion between the polymer chains and the substrate, thereby reducing friction at the wall [12-14]. Third, disentanglement of polymer chains in the bulk from the chains at the wall, i.e., cohesive slip, can also give rise to slippage $[14,15]$. Both the second and third mechanism require the shear stress to be larger than a certain critical value.

True wall slip is less commonly observed, which results from the fact that essentially all surfaces are rough at an atomic scale. It is for this reason that in most situations the no-slip boundary condition holds [16-18]. Sanchez-Reyes and Archer [19] showed for a shear-thinning polymer solution that wall roughness can be employed to suppress (the effects of) wall slip, which in general is an undesirable phenomenon in the field of rheology [10].

It is believed that slip resulting from the formation of a lubrication layer is the most important and relevant slip mechanism for most polymeric systems, in particular for dilute nonadsorbing polymer solutions $[7,9,10,20]$. The most simple representation of this mechanism is that there is a thin layer 
of a low-viscosity solvent near the wall, over which the bulk fluid with the original polymer concentration is flowing. In that case, the apparent slip length $b_{a}$ equals to

$$
b_{a}=\delta\left(\frac{\mu_{s}}{\mu_{l}}-1\right),
$$

where $\delta$ is the thickness of the depletion layer, and $\mu$ is the viscosity of the (polymer) solution $(s)$ and of the liquid in the lubrication layer $(l)$. However, as pointed out by Barnes [9], in reality the polymer concentration gradually changes between the wall and the bulk of the fluid. Tuinier and Taniguchi [21] derived for an ideal polymer solution the following expression for the slip length in the case a depletion layer is formed near a polymer-repelling wall:

$$
b_{a}=\delta \sqrt{[\mu] c_{b}} \arctan \left(\sqrt{[\mu] c_{b}}\right) .
$$

This converges to the limit of $b=\delta[\mu] c_{b}$ when $[\mu] c_{b}$ is very small. The depletion layer thickness $\delta$ approximately corresponds to the radius of gyration $R_{g}$ of the polymer in the case of a dilute solution. $[\mu]$ is the intrinsic viscosity of the solution, given in volume per mass $(\mathrm{L} / \mathrm{g})$, and $c_{b}$ is the bulk polymer concentration. Beyond the dilute regime, nonideal polymer effects can lead to slip lengths that are one order of magnitude larger than those estimated for ideal polymers solutions [22].

A commonly used polysaccharide to study non-Newtonian fluid behavior is xanthan gum. Since this is a hydrophilic and anionic biopolymer [23,24], it can be presumed to be nonadsorbing to superhydrophobic surfaces [22,24-26]. The extent of chain migration from the wall toward the bulk, and therefore the depletion layer thickness $\delta$, depends on the polymer concentration [27,28] and the shear rate [29-31]. Ausserré et al. [27] showed that for aqueous solutions of xanthan gum the depletion layer thickness $\delta$ near a nonadsorbing fused silica wall is maximum $2.2 \mathrm{~nm}$. The thickness decreases rapidly when $c>c^{*}$. The critical concentration $c^{*} \sim 1 /[\mu]$ indicates the transition of the dilute to the semidilute regime. The transition of the semidilute to the concentrated regime is denoted by $c^{* *}$. For xanthan gum, these critical concentrations are $c^{*} \sim 0.2 \mathrm{~g} / \mathrm{L}$ and $c^{* *} \sim 1 \mathrm{~g} / \mathrm{L}[32-36]$.

By evaluating Eq. (3), given that the radius of gyration $R_{g}$ is of the order of $100 \mathrm{~nm}$ for xanthan gum [37,38], we predict an apparent slip length of $b_{a} \sim 0.4 \mu \mathrm{m}$ for an aqueous $0.2 \mathrm{wt} \%$ xanthan gum solution. Compared to the typical slip lengths found in slippery, superhydrophobic systems with microscale surface structures (as for those surfaces typically $b_{f} / L \sim \mathscr{O}(1), L$ being the typical length of the surface structures [39-41]), the estimated apparent slip length is one order of magnitude smaller than the measured effective slip lengths, i.e., $b_{a}<b_{f}$. It is possible that nonideal polymer effects could increase the apparent slip length by one order of magnitude [22]. However, this effect quickly vanishes when approaching the dilute regime. Microrheometry experiments [42] and direct measurements using NMR velocimetry of the shear velocity of aqueous xanthan gum solutions up to $1 \mathrm{wt} \%$ near a PMMA or glass wall [43-45] suggest that apparent wall slip due to depletion effects is absent or otherwise very small.

Xanthan gum solutions are shear-thinning: the viscosity changes with shear rate [32-36]. The dependency of viscosity on shear may lead to some interesting behavior near textured substrates like superhydrophobic surfaces. The heterogeneity of these surfaces leads to local variations in the shear rate. For flow over superhydrophobic surfaces in the Cassie-Baxter state, i.e., when a gas is entrapped in the surface structures, the conditions at the hybrid bottom wall are a pattern of no-slip and no-shear. Various studies considering flow over a superhydrophobic surface reported large variations in the shear stress distribution at the wall [46-50]. This implies that for flows of non-Newtonian liquids (such as xanthan gum solutions) over superhydrophobic surfaces the local viscosity also varies near the wall. It immediately follows from Eq. (1) that this will affect the local and therefore the effective slip length.

The effect of a slippery wall with varying slip length on pressure-driven non-Newtonian fluid flow has been investigated analytically by Pereira [51] and by using molecular dynamics simulations by Dhondi et al. [52]. They concluded that for such surfaces, by looking at the wall-induced transverse flows, mixing is enhanced for shear-thickening fluids and suppressed for shear-thinning fluids. Applying an oscillating body force was predicted to increase transverse flow. Vayssade et al. [53] showed for aqueous suspensions of microgel particles that heterogeneity in the slip conditions at the wall has a substantial influence on the velocity profile, in particular for confined systems. Recently, Broboana et al. [54] investigated both Newtonian and non-Newtonian shear flow between patterned and liquid-filled parallel plates of a rheometer. Their results underline that surface heterogeneity leads to local variations in shear stress and viscosity, in particular for a shear-thinning liquid.

Flows of complex fluids over microscale patterned substrates have hardly been studied to date, despite the fact that non-Newtonian liquids are encountered frequently in the field of microfluidics. Examples include flows of polymer solutions [55-57], blood [58-60], and other biological samples [61] in (diagnostic) microfluidic systems. It is therefore of high relevance to understand how flows of such shear-thinning and viscoelastic solutions over microstructured surfaces behave, also in view of the potential application of superhydrophobicity for enhancing the performance of these microscale systems.

In this study, we investigate the slip flow of an inelastic non-Newtonian liquid over a superhydrophobic surface with transverse grooves and ribs - a surface often referred to as a bubble mattress. Since slip caused by wall depletion effects seems to be insignificant for aqueous xanthan gum solutions, we used these polymer solutions as a model fluid. The numerical results reveal that shear-thinning behavior can enhance effective wall slip over superhydrophobic surfaces. The maximum increase in wall slip compared to Newtonian liquids could be predicted from a theoretical analysis. We also discuss the relationship between wall slip and the hydrodynamic development of the local velocity profile.

\section{METHODOLOGY}

\section{A. Flow curves of xanthan gum solutions}

Aqueous solutions with $0.05,0.1$, and $0.2 \mathrm{wt} \%$ xanthan gum (XG) from Xanthomonas campestris (G1253, Sigma-Aldrich) 


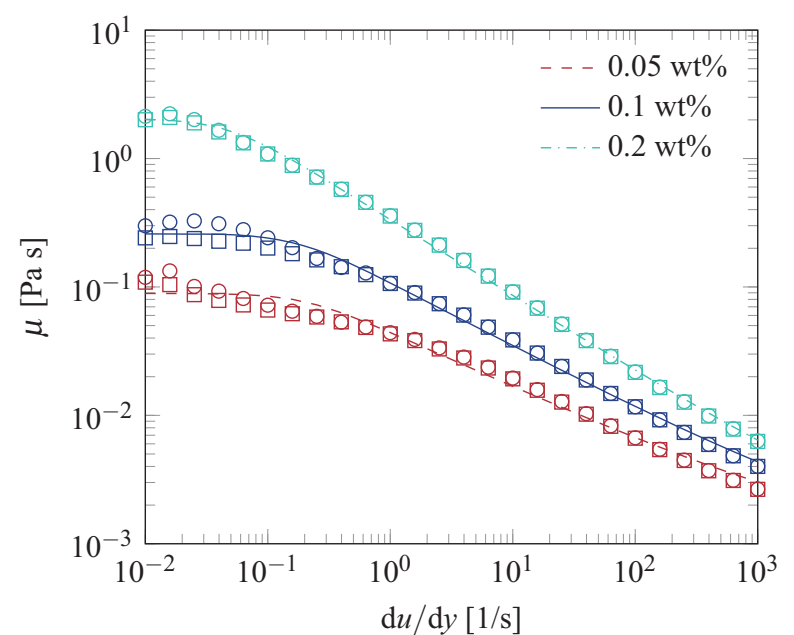

FIG. 1. Viscosity $\mu$ for multiple xanthan gum solutions. The lines are the fitted Carreau expressions to the experimental data (the circles and squares correspond to different samples).

were prepared in MilliQ water. Since $c^{*} \sim 0.2 \mathrm{~g} / \mathrm{L}$ and $c^{* *} \sim 1 \mathrm{~g} / \mathrm{L}$ [32-36], the xanthan gum concentrations $c$ of our aqueous solutions are in the semidilute regime. Viscoelastic effects are not considered in this study, which is supported by the fact that dilute xanthan gum solutions are relatively inelastic $[62,63]$. The shear-dependent viscosity of the xanthan gum solutions was characterized using an Anton Paar MCR 302 rheometer equipped with a DG26.7 double-gap concentric cylinder geometry at a temperature of $25^{\circ} \mathrm{C}$. For each xanthan gum concentration, two samples were used for the rheological experiments. The results are plotted in Fig. 1.

The flow curves are described using the Carreau expression:

$$
\mu=\mu_{\infty}+\left(\mu_{0}-\mu_{\infty}\right)\left[1+\left(\lambda \frac{\mathrm{d} u}{\mathrm{~d} y}\right)^{2}\right]^{(n-1) / 2}
$$

To determine the coefficients of this constitutive equation, the measurement data was fit in a least-squares sense using MATLAB. Fitting was performed for $\log _{10} \mu=f\left[\log _{10}(d u / d y)\right]$, with the normality of residuals checked using a normal probability plot. The fit parameters and confidence intervals are presented in Table I.

\section{B. Numerical method}

The effect of shear-thinning (nonviscoelastic) behavior on effective wall slip was investigated numerically by using a geometry with bubbles and grooves positioned perpendicular to the flow direction. The geometry, which is commonly

TABLE I. Fit parameters (95\% confidence interval) for the Carreau expression for various xanthan gum solutions. $\mu_{\infty}$ was set to the viscosity of water at $25^{\circ} \mathrm{C}$, which is $8.9 \times 10^{-4} \mathrm{~Pa} \mathrm{~s}$.

\begin{tabular}{lcrcc}
\hline \hline $\mathrm{c}[\mathrm{wt} \%]$ & $\mu_{0}[\mathrm{~Pa} \mathrm{~s}]$ & \multicolumn{1}{c}{$\lambda[\mathrm{s}]$} & $n$ & $R^{2}$ \\
\hline 0.05 & $0.0890 \pm 0.0078$ & $5.0 \pm 1.8$ & $0.563 \pm 0.024$ & 0.9855 \\
0.1 & $0.259 \pm 0.017$ & $5.9 \pm 1.4$ & $0.503 \pm 0.016$ & 0.9946 \\
0.2 & $2.05 \pm 0.12$ & $21.2 \pm 3.1$ & $0.406 \pm 0.008$ & 0.9988 \\
\hline \hline
\end{tabular}
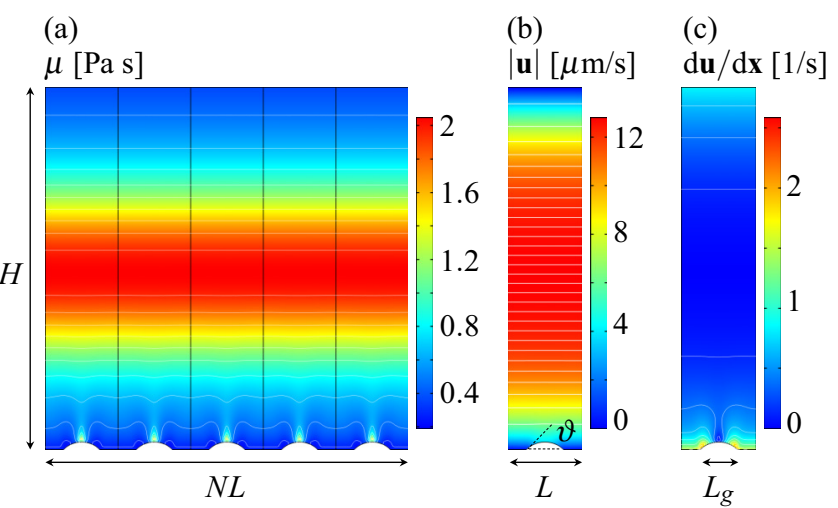

FIG. 2. Example of the periodic bubble mattress model, consisting of $N=5$ bubble units with height $H=100 \mu \mathrm{m}$, unit length $L=20 \mu \mathrm{m}$, and bubble width $L_{g}=10 \mu \mathrm{m}$. The bubble protrusion angle of, in this case, $\vartheta=45^{\circ}$ defines the gas-liquid interface curvature. The surface porosity is $\varepsilon=1 / 2$. The plot in (a) shows the local viscosity of a $0.2 \mathrm{wt} \%$ xanthan gum solution for an applied average liquid velocity of $u_{\mathrm{av}}=10^{-5} \mathrm{~m} / \mathrm{s}$. In (b) and (c) are the velocity magnitude $|\mathbf{u}|$ and the shear rate $d \mathbf{u} / d \mathbf{x}$ with $\mathbf{x}=(x, y)$ plotted for a single unit.

referred to as a bubble mattress, is illustrated in Fig. 2. The model is two-dimensional, as it is assumed that the length of the bubbles and grooves in the third dimension is very long compared to the channel height $H$. The model is also periodic, consisting of $N=5$ bubble units with a unit length $L$. The size of the bubbles $L_{g}$ is related to the surface porosity $\varepsilon=L_{g} / L$. Throughout this study, the porosity is fixed at $\varepsilon=1 / 2$. The gas-liquid interface curvature, which is known to affect the amount of wall slip $[50,64,65]$, is varied by changing the bubble protrusion angle $\vartheta$.

Fluid flow over the heterogeneously slippery surface is described by the continuity and the Navier-Stokes equation,

$$
\begin{gathered}
\nabla \cdot \mathbf{u}=0, \\
\rho(\mathbf{u} \cdot \nabla \mathbf{u})=-\nabla p+\nabla \cdot\left\{\mu\left[\nabla \mathbf{u}+(\nabla \mathbf{u})^{\mathrm{T}}\right]\right\},
\end{gathered}
$$

where $\mathbf{u}=(u, v)$. The convection terms are included in the computations, as the average velocity is sufficiently high to let $\operatorname{Re}>1$. The liquid density is assumed to be that of pure water [66], i.e., $\rho=10^{3} \mathrm{~kg} / \mathrm{m}^{3}$.

The numerical model is periodic, implying that left (source) and right (destination) boundary of the computational domain are equal in terms of velocity, i.e.,

$$
\begin{aligned}
& \mathbf{u}(0, y)=\mathbf{u}(N L, y), \\
& p(0, y)=p(N L, y) .
\end{aligned}
$$

The flow is pressure-driven, and therefore

$$
p(0, y)-p(N L, y)=-N L \nabla p .
$$

The pressure is specified at an arbitrary value and location in the domain. The pressure gradient is determined iteratively in order to fix the average velocity $u_{\mathrm{av}}$ at the specified value.

Except for the slippery bubbles, the no-slip boundary condition is applied to the upper and lower wall:

$$
\mathbf{u}=\mathbf{0} .
$$


The bubble surfaces are impermeable and perfectly slippery or shear-free, which is described by

$$
\begin{aligned}
\mathbf{n} \cdot \mathbf{u} & =0, \\
\left\{-p \mathbf{I}+\mu\left[\nabla \mathbf{u}+(\nabla \mathbf{u})^{\mathrm{T}}\right]\right\} \cdot \mathbf{n} & =\mathbf{0} .
\end{aligned}
$$

The isotropic viscosity $\mu$ of the fluid is described by the Carreau expression given in Eq. (4). This equation ignores elastic, that is time-dependent, effects. This means that the relaxation of the fluid is instantaneous and that the fluid is perfectly viscous.

The equations were solved numerically using COMSOL Multiphysics 5.2. The standard relative tolerance was $1 \times$ $10^{-3}$. P2+P1 discretization (second-order elements for velocity and first-order elements for pressure) was used to solve the Navier-Stokes equations. The mesh was refined near the walls. The complete mesh consisted of approximately 55000 domain elements and about 1200 boundary elements in the vicinity of the bubbles.

\section{Determination of the effective slip length}

After solving the numerical model, the effective slip length $b$ was determined by relating the numerically obtained average liquid velocity $u_{\text {av }}$ and pressure gradient $d p / d x$ to the slip length that would give the same average velocity and pressure gradient for a (one-sided) homogeneously slippery channel. To obtain the relationship between $u_{\mathrm{av}}, d p / d x$ and $b$ for a homogeneously slippery channel, the Navier-Stokes equation for unidirectional fluid flow is solved:

$$
0=-\frac{d p}{d x}+\frac{d}{d y}\left(\mu \frac{d u}{d y}\right) .
$$

To make the governing equation dimensionless, the following variables are introduced: $\tilde{y}=y / H, \tilde{x}=x / H, \tilde{u}=$ $u / U$, with $U=-\left(H^{2} / \mu_{0}\right)(d p / d x), \tau=\lambda U / H$, and $\tilde{\mu}=$ $\mu_{\infty} / \mu_{0}$ (note that for parallel plane Poiseuille flow $u_{\mathrm{av}}=$ $\left.-\left(H^{2} / 12 \mu\right)(d p / d x)\right)$. The dimensionless Navier-Stokes equation then becomes

$$
0=1+\frac{d}{d \tilde{y}}\left(\left\{\tilde{\mu}+(1-\tilde{\mu})\left[1+\left(\tau \frac{d \tilde{u}}{d \tilde{y}}\right)^{2}\right]^{(n-1) / 2}\right\} \frac{d \tilde{u}}{d \tilde{y}}\right) .
$$

This equation is solved by a second-order-accurate finite difference scheme combined with the MATLAB fsolve algorithm. As an initial guess for the velocity profile, the profile for Poiseuille flow with one-sided slip was used. When solving the non-Newtonian velocity profile for a range of average velocities and pressure gradients for a homogeneously slippery channel with a specified height $H$, an interpolation map $u_{\text {av }}=f(d p / d x, b)$ was obtained. Solving the COMSOL model for a specified geometry and average velocity gives the pressure gradient over the bubble mattress. Subsequently the interpolation map was used to determine the effective slip length for Carreau flow over the bubble mattress by interpolation using the MATLAB function interp2 (interpolation based on a cubic spline).

\section{LIMITING VALUES FOR EFFECTIVE WALL SLIP}

In this section, we derive the theoretical limit for the effective slip length for non-Newtonian flow over a heterogeneously slippery surface consisting of an alternating pattern of no-slip and no-shear regions positioned perpendicular to the flow direction. Although the shear-thinning behavior of fluids like xanthan gum solutions is well described by constitutive relations that also capture the zero-shear and infinite-shear Newtonian plateaus, the shear-thinning region is often described by a power law:

$$
\mu=\mu_{0}\left(\frac{\partial u}{\partial r}\right)^{n-1}
$$

Because of its mathematical simplicity, we will describe the derivation of the theoretical limit for effective wall slip for a power-law fluid. For the same reason, we change from a planar to a cylindrical coordinate system [see Fig. 3(a) for an illustration of the cylindrical system]. The derived equations will be analogous for a planar geometry and can therefore also be used to predict approximately the maximum slip length for the planar system shown in Fig. 2 (as we will demonstrate later in Sec. IV B).

A schematic of the system is given in Fig. 3(b). Here, we can distinguish four different regions with a length $\tilde{l}_{i}=l_{i} / R$, with $i=[1 \ldots 4]$. As indicated in Fig. 3, in the regions 1 and 3 the flow is developing and therefore crosses the noslip region $\tilde{L}_{1}=L_{1} / R$ with $\tilde{b}_{1}=0$ and the no-shear region $\tilde{L}_{2}$ with $\tilde{b}_{2}=\infty$. The total length of a no-slip/no-shear unit

(a)
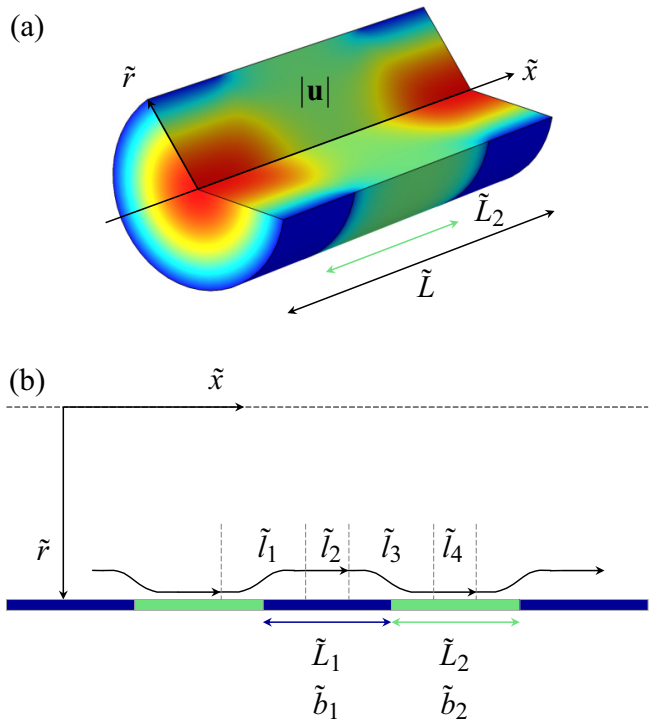

FIG. 3. A cylindrical geometry is used to investigate the relationship between the effective slip length $\tilde{b}=b / R$ and the relative bubble unit confinement $\tilde{L}=L / R$. In (a), the velocity magnitude $|\mathbf{u}|$ for $\tilde{L}=5.0, n=1$, and $\varepsilon=1 / 2$ is shown. The color scale varies from red for $|\mathbf{u}|=2$ to dark blue for $|\mathbf{u}|=0$. In (b), a schematic of the system is given. The flow pattern over the no-slip ( $\tilde{L}_{1}$ with $\left.\tilde{b}_{1}=0\right)$ and no-shear regions ( $\tilde{L}_{2}$ with $\left.\tilde{b}_{2}=\infty\right)$ can be divided into four different subregions: $\tilde{l}_{i}$ with $i=[1 \ldots 4]$. When $\tilde{L} \rightarrow \infty$, the effective pressure drop in the domain is dominated by the no-slip region $\tilde{l}_{2}$. 
equals $\tilde{L}=\tilde{L}_{1}+\tilde{L}_{2}$. In the case the flow over these regions is not fully developed, we find that $\tilde{l}_{2}, \tilde{l}_{4}=0$. Note that, since we are considering Stokes flow, $\tilde{l}_{1}=\tilde{l}_{3}$. On the other hand, when the no-slip and no-shear regions would be infinitely long compared to the tube radius, i.e., $\tilde{L}_{1}, \tilde{L}_{2} \rightarrow \infty$, we find that $\left(\tilde{l}_{1}, \tilde{l}_{3}\right) /\left(\tilde{l}_{2}, \tilde{l}_{4}\right) \rightarrow 0$. The flow is hydrodynamically fully developed and has completely adapted to the local boundary conditions.

In order to obtain the effective slip length for this system, one cannot take the spatial average of the local slip length, as this will yield an infinite slip length: $\tilde{b}_{f} \neq\left(\tilde{L}_{1} \tilde{b}_{1}+\right.$ $\left.\tilde{L}_{2} \tilde{b}_{2}\right) /\left(\tilde{L}_{1}+\tilde{L}_{2}\right)=\infty$. However, one can define an effective pressure gradient, which subsequently can be used to obtain the effective slip length:

$$
\left(\frac{\partial \tilde{p}}{\partial \tilde{x}}\right)_{f}=\sum_{i=1}^{4} \tilde{l}_{i}\left(\frac{\partial \tilde{p}}{\partial \tilde{x}}\right)_{i} / \sum_{i=1}^{n} \tilde{l}_{i} .
$$

In the case of a developing flow, i.e., $\tilde{l}_{2}, \tilde{l}_{4}=0$, this equation cannot be evaluated a priori, since the pressure drop over the regions 1 and 3 is not known. However, when $\tilde{L}_{1}, \tilde{L}_{2} \rightarrow \infty$ and thus $\sum \tilde{l}_{i} \rightarrow \tilde{l}_{2}+\tilde{l}_{4}$, we find that

$$
\left(\frac{\partial \tilde{p}}{\partial \tilde{x}}\right)_{f}=\frac{1}{\tilde{l}_{2}+\tilde{l}_{4}}\left[\tilde{l}_{2}\left(\frac{\partial \tilde{p}}{\partial \tilde{x}}\right)_{2}+\tilde{l}_{4}\left(\frac{\partial \tilde{p}}{\partial \tilde{x}}\right)_{4}\right] .
$$

Considering that the local pressure gradient for a no-shear region equals zero and that the surface porosity can be approximated by $\varepsilon=\tilde{L}_{2} /\left(\tilde{L}_{1}+\tilde{L}_{2}\right) \sim \tilde{l}_{4} /\left(\tilde{l}_{2}+\tilde{l}_{4}\right)$, this finally yields the following expression for the effective pressure gradient:

$$
\left(\frac{\partial \tilde{p}}{\partial \tilde{x}}\right)_{f}=\frac{\tilde{l}_{2}}{\tilde{l}_{2}+\tilde{l}_{4}}\left(\frac{\partial \tilde{p}}{\partial \tilde{x}}\right)_{2}=(1-\varepsilon)\left(\frac{\partial \tilde{p}}{\partial \tilde{x}}\right)_{2} .
$$

The pressure gradient for the flow of a power-law fluid through a nonslippery tube can be evaluated, and thus an expression can be found for the maximum slip length that can be achieved.

The velocity profile of a power-law fluid through a tube with a slippery wall having a uniform slip length $b$ and a pressure drop $\partial p / \partial x$ is given by

$$
u=R\left(-\frac{R}{2 \mu_{0}} \frac{\partial p}{\partial x}\right)^{1 / n}\left\{\frac{1}{1 / n+1}\left[1-\left(\frac{r}{R}\right)^{1 / n+1}\right]+\frac{b}{R}\right\} .
$$

The average velocity then equals

$$
u_{\mathrm{av}}=R\left(-\frac{R}{2 \mu_{0}} \frac{\partial p}{\partial x}\right)^{1 / n}\left[\frac{1}{1 / n+3}+\frac{b}{R}\right] .
$$

Both equations can be made dimensionless using $\quad b=\tilde{b} R, x=\tilde{x} R, u=\tilde{u} u_{\mathrm{av}}, \quad$ and $\quad \partial p / \partial x=$ $\left(R / \mu_{0}\right)\left(R / u_{\mathrm{av}}\right)^{n}(\partial \tilde{p} / \partial \tilde{x})$, yielding

$$
\tilde{u}=\left(-\frac{1}{2} \frac{\partial \tilde{p}}{\partial \tilde{x}}\right)^{1 / n}\left[\frac{1}{1 / n+1}\left(1-\tilde{r}^{1 / n+1}\right)+\tilde{b}\right]
$$

for the velocity profile, and

$$
\tilde{u}_{\mathrm{av}}=1=\left(-\frac{1}{2} \frac{\partial \tilde{p}}{\partial \tilde{x}}\right)^{1 / n}\left[\frac{1}{1 / n+3}+\tilde{b}\right]
$$

for the average dimensionless velocity, which equals one. Thus, for a system with an average velocity $\tilde{u}_{\mathrm{av}}=1$ having a known pressure gradient, we can calculate the slip length by

$$
\tilde{b}=\left(-\frac{1}{2} \frac{\partial \tilde{p}}{\partial \tilde{x}}\right)^{-1 / n}-\frac{1}{1 / n+3} .
$$

We can now evaluate the maximum slip length for a power-law flow through a tube with a heterogeneously slippery wall. As expressed by Eq. (16), when $\tilde{L} \rightarrow \infty$, the effective pressure gradient is fully dominated by the gradient over the no-slip regions. This gives, when substituting Eq. (20) for $\tilde{b}=0$ into Eq. (16), the following relation for the effective pressure gradient:

$$
\left(\frac{\partial \tilde{p}}{\partial \tilde{x}}\right)_{f}=-2(1-\varepsilon)\left(\frac{1}{n}+3\right)^{n} .
$$

When using this expression for the effective pressure gradient and substituting that into Eq. (21), we finally obtain the following expression for the maximum effective slip length $\tilde{b}_{\max }$ when $\tilde{L} \rightarrow \infty$ :

$$
\begin{aligned}
\tilde{b}_{\max } & =\frac{1}{(1 / n+3)(1-\varepsilon)^{1 / n}}-\frac{1}{1 / n+3} \\
& =\frac{1-(1-\varepsilon)^{1 / n}}{(1 / n+3)(1-\varepsilon)^{1 / n}} .
\end{aligned}
$$

In the case of a Newtonian fluid with $n=1$, this yields

$$
\tilde{b}_{\max }=\frac{1}{4} \frac{\varepsilon}{1-\varepsilon} .
$$

Equation (24) for a Newtonian fluid has also been derived by Lauga and Stone [40], although they obtained this result by solving the flow field analytically and looking in the asymptotic limit for large distances between the slip regions.

These results are confirmed numerically using COMSOL Multiphysics 5.2 using a periodic cylindrical model consisting of one repeating unit of a transverse no-slip and no-shear region (the no-shear region can be considered as a flat gas bubble with $\vartheta=0^{\circ}$ ). An example of the model is shown in Fig. 3(a) for $\tilde{L}=5.0$.

\section{RESULTS AND DISCUSSION}

\section{A. Numerical results}

In this section, we describe and discuss our numerical results. The viscosity of the aqueous xanthan gum solutions is described by the Carreau expression. In Fig. 4, the effective slip length $b$ for a $0.2 \mathrm{wt} \%$ xanthan gum solution is plotted as a function of the bubble protrusion angle $\vartheta$ for various imposed average velocities. The slip length is normalized by the bubble width $L_{g}$. For small flow rates, i.e., $u_{\mathrm{av}} \leqslant 10^{-7} \mathrm{~m} / \mathrm{s}$, we observe that the slip-length profile coincides with that of water. When increasing the velocity, for all protrusion angles the slip length increases and reaches a maximum when $u_{\mathrm{av}}=10^{-5} \mathrm{~m} / \mathrm{s}$. For larger liquid velocities, this decreases again and converges toward the profile for water. Note that here for liquid velocities larger than $10^{1} \mathrm{~m} / \mathrm{s}$, inertial effects become significant. For that reason, the slip length profile will approach but not fully overlap with that of water (which also starts to change when $u_{\mathrm{av}} \gg 1 \mathrm{~m} / \mathrm{s}$ ). 


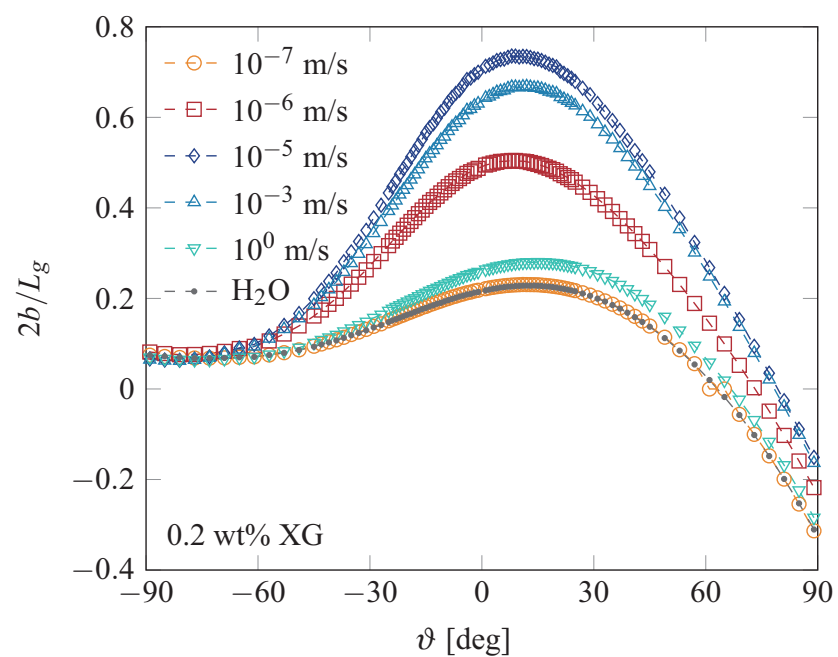

FIG. 4. Dimensionless effective slip length $2 b / L_{g}$ for a $0.2 \mathrm{wt} \%$ xanthan gum $(\mathrm{XG})$ solution as a function of the bubble protrusion angle $\vartheta$ for various average liquid velocities $u_{\mathrm{av}}\left(H=100 \mu \mathrm{m}, L_{g}=\right.$ $10 \mu \mathrm{m}, \varepsilon=1 / 2$ ). A liquid velocity of $u_{\mathrm{av}}=10^{-5} \mathrm{~m} / \mathrm{s}$ and a viscosity of $\mu=0.89 \mathrm{mPa}$ s was used to calculate the slip length profile for water.

This dependency of the slip length on the velocity can be related to the shear-thinning properties of the modeled xanthan gum solution (see Fig. 1). In Fig. 5(a), the slip length $b$ is plotted as a function of the average velocity $u_{\mathrm{av}}$ for $\vartheta=1^{\circ}$. For very small flow rates, the shear rates in the fluid flow are very small and the viscosity equals the zero-shear viscosity: $\mu=\mu_{0}$. For intermediate flow rates, the system is operated in the shear-thinning regime of the xanthan gum solutions and the viscosity decreases with increasing flow rates and, consequently, increasing shear rates. As illustrated by Fig. 2(a), in this regime the viscosity near the heterogeneously slippery wall strongly varies with position. When the shear rate approaches the upper Newtonian plateau, $d u / d y>10^{2}$ $1 / \mathrm{s}$, the decrease in viscosity with increasing shear rate levels off and the viscosity approaches the infinite shear viscosity: $\mu \rightarrow \mu_{\infty}$.

Note that the critical protrusion angle, the angle for which wall slip becomes negative, also increases when operating the system in the shear-thinning region. It shifts from $63^{\circ}$ for the zero-shear Newtonian plateau to a maximum value of $79^{\circ}$ for $u_{\mathrm{av}}=10^{-5} \mathrm{~m} / \mathrm{s}$. The increase in slip length with flow rate is considerably smaller for very large positive protrusion angles than for flat bubbles. In the case of highly negative angles, the liquid protrudes in the grooves and there is no enhancement. The liquid near the bubble surface is almost stagnant, for which reason the viscosity near the shear-free gas-liquid boundary also is very high: $\mu \sim \mu_{0}$.

We therefore conclude that when the system is operated in one of the Newtonian plateaus, the slip length profile resembles the profile for water. Preliminary experimental results, which are shown in the Appendix, also confirm this: for very high flow rates $\left(u_{\mathrm{av}}=10^{-1} \mathrm{~m} / \mathrm{s}\right)$ the effective slip lengths for the various xanthan gum solutions overlap with those for water. These results also suggest that slip resulting from any depletion related effects is insignificant when compared with the slip induced by the superhydrophobic surface.
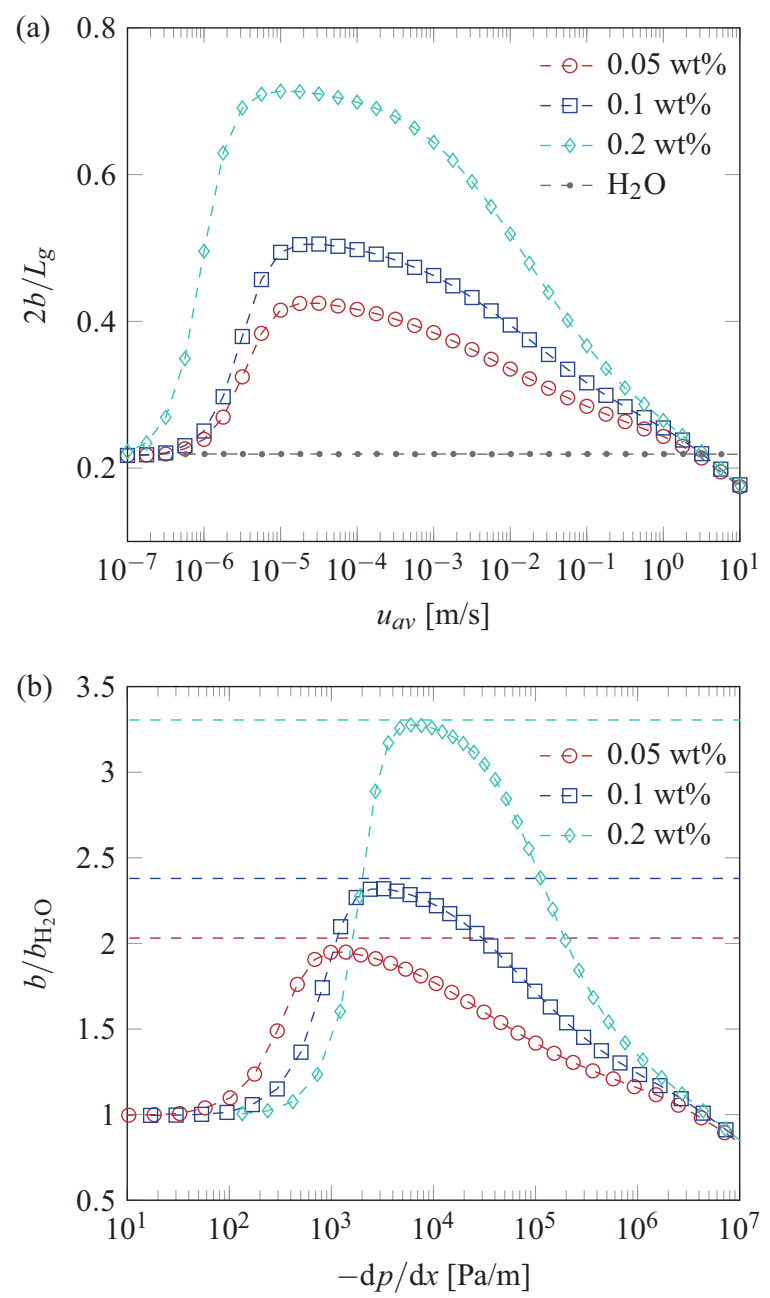

FIG. 5. In (a) the effective slip length $2 b / L_{g}$ is plotted as a function of the average liquid velocity $u_{\mathrm{av}}$ for a protrusion angle of $\vartheta=1^{\circ}$ for various xanthan gum solutions. In (b), the slip enhancement factor $b / b_{\mathrm{H}_{2} \mathrm{O}}$ for $\vartheta=1^{\circ}$ is plotted as a function of the effective pressure gradient $d p / d x$, where $b_{\mathrm{H}_{2} \mathrm{O}}$ has been determined for $u_{\mathrm{av}}=10^{-5} \mathrm{~m} / \mathrm{s}$. For both (a) and (b), $H=100 \mu \mathrm{m}, L_{g}=10 \mu \mathrm{m}$, and $\varepsilon=1 / 2$. The horizontal dashed lines in (b) are the analytically predicted enhancement factors $\tilde{b}_{\max }(n) / \tilde{b}_{\max }(1)$ as obtained from Eq. (23).

In the shear-thinning region, the slip length increases with increasing flow rate. Despite the fact that, as follows from Eq. (1), the slip length is a function of both viscosity and shear rate at the wall, in this region $\mu \propto\left(d_{y} u\right)^{n-1}$ and the viscosity therefore decreases faster than the shear rate increases when $n<1$. Assuming that the friction factor $k$ is constant, this implies that wall slip should increase. This is confirmed by the numerical results.

The enhancement in slip length $b / b_{\mathrm{H}_{2} \mathrm{O}}$, i.e., the slip length for the non-Newtonian xanthan gum solution over that for water, is plotted in Fig. 5(b). The more shear-thinning the xanthan gum solutions are (i.e., the smaller $n$ is), the larger the enhancement. For a $0.2 \mathrm{wt} \%$ xanthan gum solution with $n=$ 0.406 , a maximum enhancement factor of 3.2 can be achieved. Note the nonlinear relationship between the average velocity and the driving force when comparing Figs. 5(a) and 5(b): $u_{\mathrm{av}}$ 
is not proportional to $d p / d x$. Because of the shear-thinning properties of the fluid, the flow rate increases faster than the applied pressure gradient.

The results described in this section underline that it is important that the constitutive equation properly describes the rheological behavior of the fluid for very small or very large shear rates. A constitutive equation like the Carreau expression does this; modeling the liquid as a power-law fluid would have predicted an enhancement in slip for all flow/shear rates [67]. However, when operating in the shear-thinning regime with shear rates in the range of roughly $10^{-1}<d u / d y<10^{3} 1 / \mathrm{s}$, the power-law does predict the correct behavior.

\section{B. Predicting the enhancement}

In Sec. III we have derived an expression that gives the maximum slip length that can be obtained when the bubbles become infinitely large compared to the typical system size, i.e., when $\tilde{L}=L / R \rightarrow \infty$. The results presented above, however, are for a bubble mattress with $H=100 \mu \mathrm{m}, L_{g}=$ $10 \mu \mathrm{m}$, and $\varepsilon=1 / 2$. This only gives a confinement factor of $L / H=0.2$.
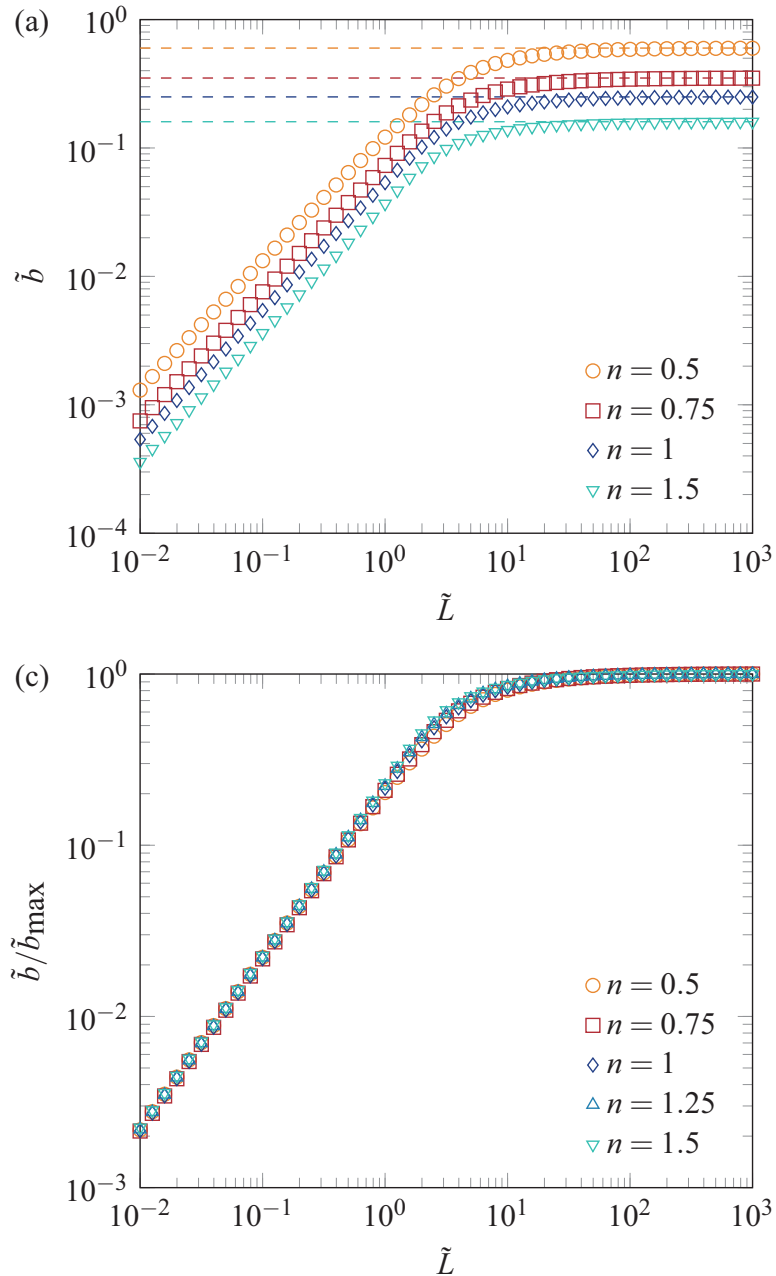

To investigate how the amount of wall slip changes with increasing confinement, we have simulated fluid flow through a tube with a pattern of transverse no-slip and no-shear regions. This can be interpreted as a cylindrical bubble mattress with flat bubbles $\left(\vartheta=0^{\circ}\right)$. The surface area ratio of no-slip to no-shear region equals $\varepsilon=1 / 2$. The results are plotted in Fig. 6(a). For $\tilde{L}<10^{0}$, where the slope of the log-log plot equals one, the effective slip length $\tilde{b}$ grows linearly with the confinement $\tilde{L}$. As will be discussed in Sec. IV C, for $\tilde{L}<10^{0}$ the flow profile cannot fully adapt to either the no-slip or no-shear condition at the wall and is therefore still hydrodynamically developing. The slip length reaches its maximum when $\tilde{L}>10^{2}$, showing a perfect overlap with the analytically calculated values given by Eq. (23).

Note that in Fig. 6(a) the slip length is nondimensionalized using the tube radius $R$, whereas in Figs. 4 and 5 the slip length is nondimensionalized based on the bubble width $L_{g}$. These are related to each other, as

$$
\frac{2 b}{L_{g}}=\frac{2 \tilde{b}}{\varepsilon \tilde{L}} .
$$
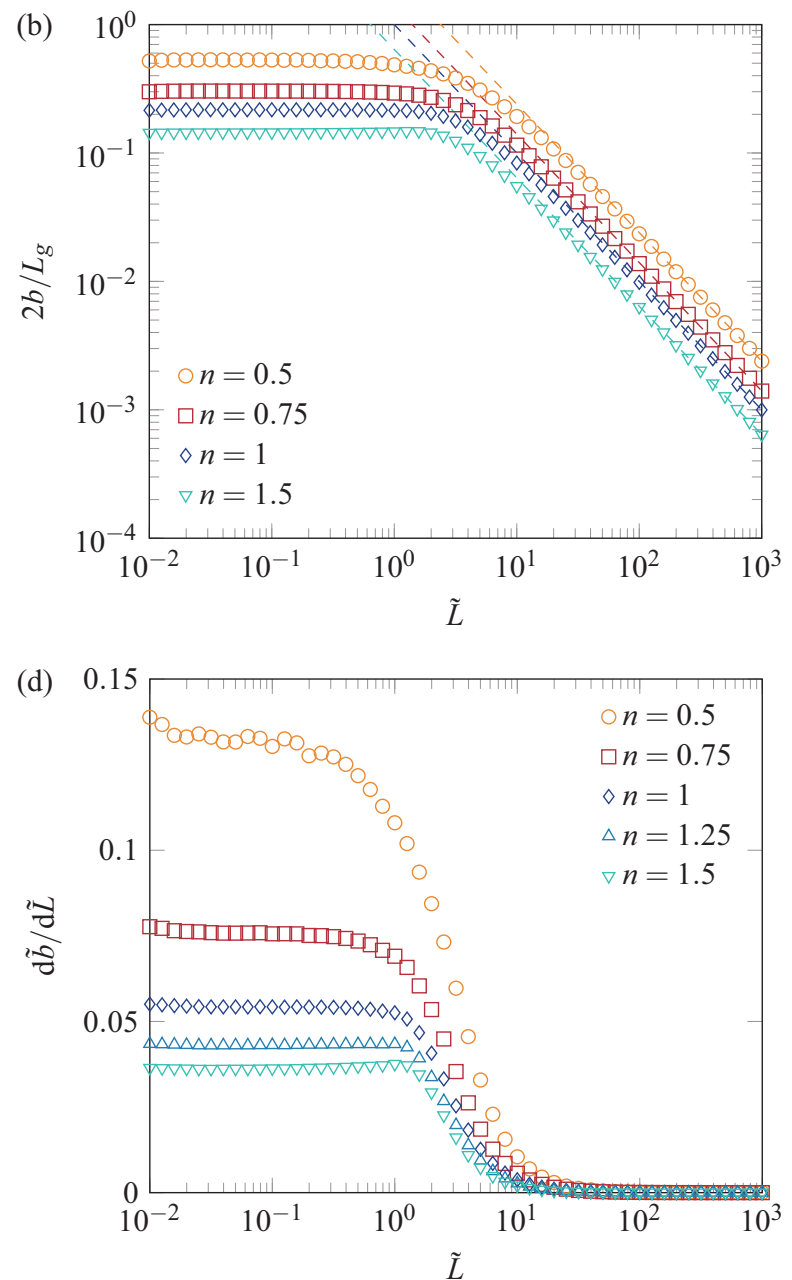

FIG. 6. In (a) the effective slip length $\tilde{b}=b / R$ is plotted as a function of the no-slip/no-shear unit length $\tilde{L}=L / R$ for various power-law indices $n$. The same data is replotted in (b), showing now the slip length normalised with the length of the no-shear region $L_{g}$. The dashed lines in (a) and (b) are the theoretical maxima for $\tilde{b}$ as calculated using Eq. (23). When normalizing $\tilde{b}$ using the maximum effective slip length $\tilde{b}_{\text {max }}$, the master curve shown in (c) is obtained. In (d) the slope $d \tilde{b} / d \tilde{L}$ of the slip length profiles shown in (a) is plotted. For all figures, $\varepsilon=1 / 2$. 


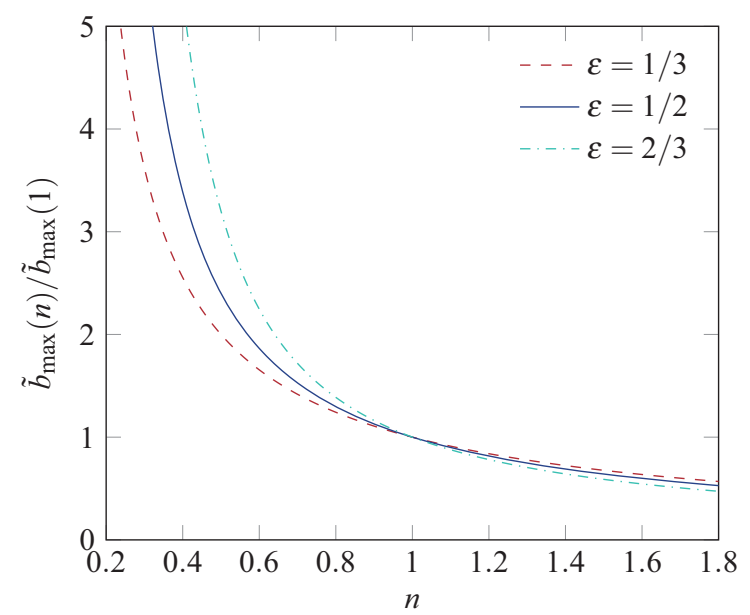

FIG. 7. Wall-slip enhancement factor $\tilde{b}_{\max }(n) / \tilde{b}_{\max }(1)$, given by Eq. (23), as a function of the power-law index $n$ for three different surface porosities $\varepsilon$.

This implies that plotting $2 b / L_{g}(\tilde{L})$ instead of $\tilde{b}(\tilde{L})$ will reduce the slope of the profiles in Fig. 6(a) by one. Figure 6(b) shows that this indeed is the case. For $\tilde{L}<10^{0}, 2 b / L_{g}$ is constant, whereas this decreases linearly for $\tilde{L}>10^{2}$.

Two limiting regimes can therefore be identified. In the first regime, the bubble confinement is very small $(\tilde{L} \ll 1)$ and $2 b / L g$ is constant. The slip length is "suboptimal" in the sense that it is smaller than the maximum slip possible for the set of wall conditions. The slip length grows linearly with the length of the no-shear region $\varepsilon \tilde{L}$, which can be interpreted as the bubble width. In the second regime, the bubble confinement is very large $(\tilde{L} \gg 1)$ and $\tilde{b}$ is constant. The slip length reaches its maximum value, i.e., $\tilde{b}=\tilde{b}_{\max }$, and the effective pressure gradient is constant, because it is fully dominated by the pressure drop over the no-slip region, which grows linearly with the length $(1-\varepsilon) \tilde{L}$ of this region.

Figure 6(c) illustrates that the expression for maximum wall slip, Eq. (23), can also be used to predict the enhancement in the regime $\tilde{L}<10^{0}$ where $2 b / L_{g}$ is constant. All profiles for $\tilde{b}$ collapse onto a master curve when normalizing them by the corresponding maximum slip length $\tilde{b}_{\max }$. This indicates that the ratio in $\tilde{b}_{\max }$ for power-law fluids having different exponents $n$ remains constant for all $\tilde{L}$, except for the transition regime $10^{0}<\tilde{L}<10^{2}$.

The slope of the profiles must be different for varying power-law indices $n$, since $\tilde{b} \rightarrow 0$ when $\tilde{L} \rightarrow 0$. The effective slip length needs to grow faster for a shear-thinning than for a shear-thickening liquid, as all power-law fluids reach $\tilde{b}_{\max }$ for approximately the same value of $\tilde{L}$. Figure 6(d) supports this conclusion. Note that the slope of the curves not only changes with $n$, but also with the surface porosity $\varepsilon$. We found that the slope approximately scales as $d \tilde{b} / d \tilde{L} \propto 1 / n$ and $d \tilde{b} / d \tilde{L} \propto \varepsilon /(1-\varepsilon)$.

The maximum slip length $\tilde{b}_{\max }$ predicted by Eq. (23) is plotted in Fig. 7 as a function of the power-law index $n$ for three different surface porosities $\varepsilon$. The curves, which have been normalized by $\tilde{b}_{\max }$ for a Newtonian fluid with $n=1$, give the slip enhancement factor for a given $n$ and $\varepsilon$. Shear-thinning behavior $(n<1)$ increases relative wall slip, whereas shear thickening behavior $(n>1)$ reduces it. Wall slip increases with $\varepsilon$ for shear-thinning liquids with $n<1$, whereas it decreases with $\varepsilon$ for shear-thickening fluids with $n>1$.

We found that Eq. (23) can also be used to predict the maximum slip length in the regime $\tilde{L}<10^{0}$ where $2 b / L_{g}$ is constant. For that reason, we plotted in Fig. 5(b) the analytically predicted enhancement factors for the three different xanthan gum solutions. The horizontal dashed lines in Fig. 5(b), which represent the analytical values, indeed show very good agreement with the numerically calculated maximum enhancement factors.

\section{Relationship to hydrodynamic development length}

Figure 6(a) shows that for $\tilde{L}<10^{\circ}$, the effective slip length $\tilde{b}$ grows linearly with $\tilde{L}$. This can be related to the hydrodynamic development of the velocity profile. The length $L_{d}$ required for full development, also called the entrance length, is given by [68]

$$
\frac{L_{d}}{D}=0.6+0.056 \mathrm{Re},
$$

which in the case of Stokes flow with $\operatorname{Re} \rightarrow 0$ and using the radius $R$ instead of the diameter $D$ reduces to

$$
\frac{L_{d}}{R}=1.2 \text {. }
$$

Considering the flow over the no-slip and no-shear regions, illustrated in Fig. 8(a), we observe that in each region the flow is developing twice: when entering and when exiting the region. Therefore, we expect the profile to be fully developed when

$$
\begin{aligned}
\varepsilon \tilde{L}>2.4 & \text { when } \varepsilon \leqslant 1 / 2, \\
(1-\varepsilon) \tilde{L}>2.4 & \text { when } \varepsilon>1 / 2 .
\end{aligned}
$$

This is an estimation, as in the model the flow is already partly developed when entering the region. Equation (26) is based on a system where at the start of a no-slip region the velocity profile is uniform (which, in the case of Stokes flow as considered here, is equivalent to a system with a parabolic velocity profile at the start of a no-shear region). Note that the entrance length only changes slightly for an inelastic nonNewtonian fluid [69].

The development for a Newtonian liquid, i.e., $n=1$, is also illustrated in Fig. 8(a). When $\tilde{L}=5.6$, we see that the profile in the middle of the domain is uniform. This is also confirmed in Fig. 8(b), which shows that for this domain length the velocity at the wall essentially reaches its maximum possible value: $\left.\tilde{u}\right|_{\tilde{r}=1}=1$. The results shown in Fig. 8 are therefore in agreement with Eq. (28), which predicts that for this system the flow is hydrodynamically developed when $\tilde{L}>4.8$.

The slip length $\tilde{b}$, see Fig. 6(a), does not increase linearly with $\tilde{L}$ anymore when $\varepsilon \tilde{L}>2.4$. It requires $\tilde{L}$ to be roughly two orders of magnitude larger, however, to let the slip length reach the actual maximum value $\tilde{b}_{\max }$. In the transition region $10^{0}<\tilde{L}<10^{2}$ the development regions $\tilde{l}_{1}$ and $\tilde{l}_{3}$, see Figs. 3(b) and 8(a), still contribute to the overall or effective pressure gradient over the system. This gradient slowly converges to the pressure drop over the nonslippery 
(a) $\tilde{L}$
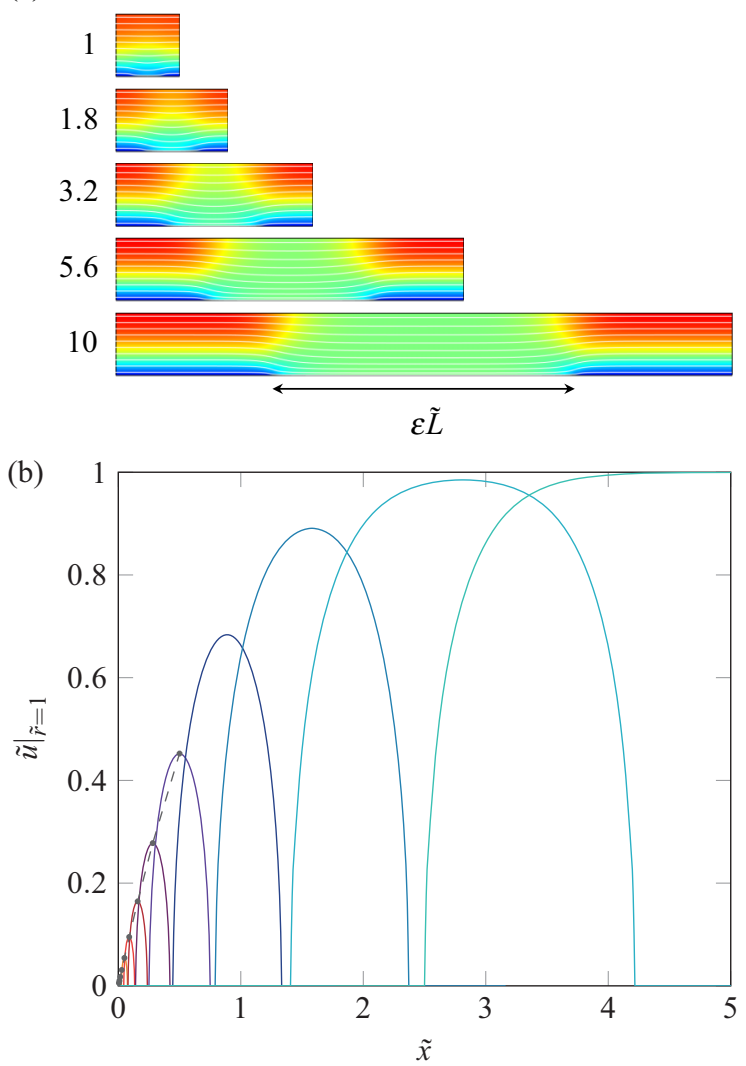

FIG. 8. As long as the flow is hydrodynamically developing, the slip length $\tilde{b}$ grows linearly with the unit length $\tilde{L}$. (a) For $\varepsilon \tilde{L}>2.4$, the flow becomes hydrodynamically developed, here shown for a Newtonian liquid with $n=1$. (b) The liquid velocity at the no-shear wall then also reaches the maximum possible value of $\left.\tilde{u}\right|_{\tilde{r}=1}=1$ at, in this case, $\tilde{L} / 2$. Here, the velocity profiles are plotted for $\tilde{L}=$ $10^{-2+0.25 k}$ with $k=[0 \ldots 12], n=1$, and $\varepsilon=1 / 2$. The increase in the maximum liquid velocity at the wall with $\tilde{L}$ for different power law indices $n$ (here represented for $n=1$ by the gray dashed curve for $\tilde{L} \leqslant 1$ ) is correlated with $d \tilde{b} / d \tilde{L}$ as plotted in Fig. 6(d), showing the same behavior.

region, wherefore ultimately the conditions are reached for which the expression for $\tilde{b}_{\max }$, Eq. (23), is valid.

\section{CONCLUSION}

In this study, we have investigated the flow of inelastic, non-Newtonian fluids over heterogeneously slippery superhydrophobic surfaces. The numerical and analytical results show that wall slip is increased for shear-thinning liquids, whereas shear-thickening fluids result in reduced slippage. In the case of shear-thinning liquids, for which we took aqueous xanthan gum solutions as a model fluid, the slip enhancement with respect to water strongly depends on the applied driving force. Only in the shear-thinning regime, wall slip can be enhanced. The slip length converges to that of water when the system is operated in one of the Newtonian regimes.

The maximum slip enhancement could be predicted analytically for a cylindrical geometry. The predicted enhancement showed good agreement with the numerical results for a planar geometry. This indicates that the theoretical result is also applicable to other geometries. Wall slip is maximized when the bubbles are much larger than the characteristic dimension of the system: the flow over the heterogeneous surface can then fully develop according to the hydrodynamic
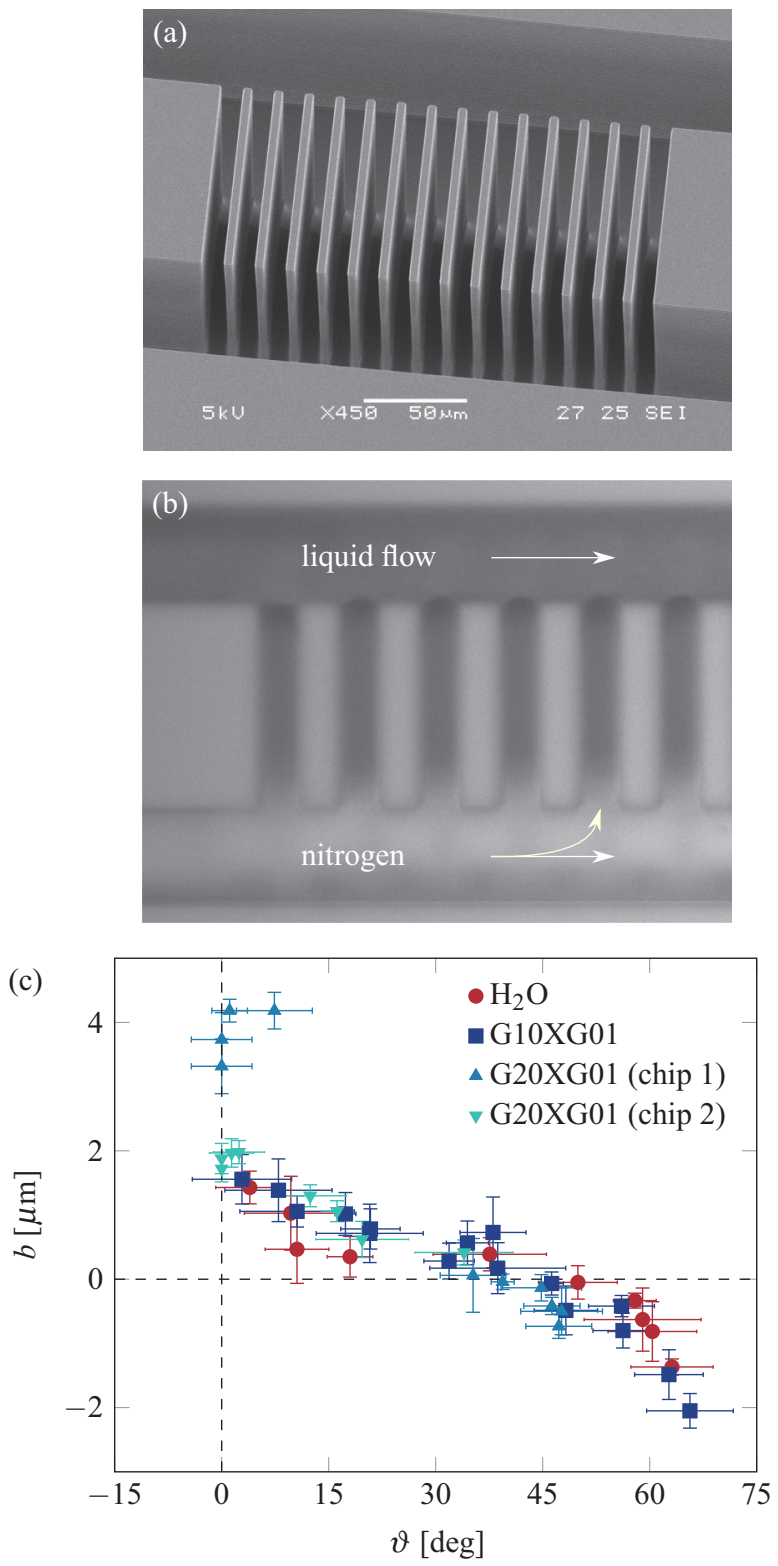

FIG. 9. In (a), a SEM image is shown of one of the microfluidic devices that were used to study non-Newtonian flow over a bubble mattress. They consist of two main channels, connected to each other by 15 microchannels. Because the wall was hydrophobized, as shown in (b), the side channels were filled with a gas (nitrogen) and the liquid in the upper channel was flowing over an array of no-slip wall segments and gas bubbles. Here, the image was focused at the midplane of the channel, where also the $\mu \mathrm{PIV}$ experiments were performed. In (c), for a chip with $H=50 \mu \mathrm{m}, L_{g}=10 \mu \mathrm{m}$, and $\varepsilon=2 / 3$, the effective slip lengths $b$ are plotted as a function of the bubble protrusion angle $\vartheta$ for water and for shear-thinning solutions of $0.1 \mathrm{wt} \%$ xanthan gum (XG) dissolved in a mixture of water and either 10 or $20 \mathrm{wt} \%$ glycerol (G). 
boundary conditions. Although in practice it is hard to achieve such conditions (the slip regions are highly confined), the enhancement factor as derived analytically from the maximum slip length remains constant for all confinement factors.

Experiments are required to confirm the predicted increase in wall slip for flow of inelastic shear-thinning liquids over heterogeneously slippery surfaces. Aqueous xanthan gum solutions appear to be suitable for this purpose, since the preliminary experimental results indicate that for these solutions slip induced by depletion effects at the wall is either absent or negligible compared to slip arising from superhydrophobicity. The experiments are challenging in terms of stability and controllability, as stable non-Newtonian fluid flows over microscale, heterogeneously slippery surfaces need to be established at low flow rates. However, from both a fundamental and an application point of view, they are of high value to explore the potential of superhydrophobic slip enhancement for non-Newtonian fluid flow.

\section{ACKNOWLEDGMENTS}

R.G.H.L. acknowledges the European Research Council for the ERC Starting Grant No. 307342-TRAM.

\section{APPENDIX: PRELIMINARY EXPERIMENTAL RESULTS}

Experimentally, wall slip for non-Newtonian fluid flow was investigated using microparticle image velocimetry ( $\mu$ PIV) [67]. For this purpose, glass-silicon microfluidic devices were fabricated using standard photolithography and deep reactive ion etching. The structured silicon wafers were anodically bonded to glass wafers. The silicon microchannels, which were all $100 \mu \mathrm{m}$ deep, had different main channel heights $H$, side channel widths $L_{g}$, and surface porosities $\varepsilon$ (see Fig. 9(a) for an example). The channels were hydrophobized using gaseous chlorotrimethylsilane (Sigma-Aldrich).

For the $\mu$ PIV experiments, a Litron LDY301 dual cavity $\mathrm{Nd}$ :YLF laser with a wavelength of $527 \mathrm{~nm}$ connected to an inverted Zeiss Axio Observer.Z1 microscope was used. The microscope was equipped with a LaVision Imager intense $670 \mathrm{KD}$ double shutter camera. The experiments were performed under stationary flow with an average liquid velocity of $u_{\mathrm{av}}=10^{-1} \mathrm{~m} / \mathrm{s}$ using a Bronkhorst mass flow controller; see Fig. 9(b). A Bronkhorst pressure controller was used to regulate the gas pressure. Various xanthan gum (G1253, Sigma-Aldrich) solutions in a mixture of glycerol (Sigma-Aldrich) and water were used, in which $0.01 \mathrm{wt} \%$ red fluorescing polystyrene particles (microParticles, Germany) with a diameter of $0.5 \mu \mathrm{m}$ were dispersed. To determine the flow field, required to evaluate the effective slip length, 100 image pairs were captured at a $63 \times$ magnification (with a numerical aperture of 0.75 ). The time difference between frame $\mathrm{A}$ and $\mathrm{B}$ of each image pair was set to $6 \mu \mathrm{s}$, which gave an optimal average particle shift of about 5 pixels. The images were preprocessed by removal of the background intensity and the out-of-focus particles. The occurrence of spurious vectors in the vector fields was suppressed by using a geometric mask. The vector fields were obtained using the DaVis 8 PIV software package by a multipass calculation, starting with two passes using an interrogation window of $64 \times 64$ pixels and finishing with an interrogation window of $16 \times 16$ pixels, all with a $50 \%$ window overlap. A sliding sum of correlation was employed, which uses five consecutive image pairs for the cross correlation to artificially increase the particle density.

The local slip lengths were determined by extrapolation of the local velocity profile using the six data points adjacent to the wall. Averaging of these local slip lengths gave the effective slip length.

The experimentally determined effective slip lengths are plotted in Fig. 9(c) for a chip with $H=50 \mu \mathrm{m}, L_{g}=10 \mu \mathrm{m}$, and $\varepsilon=2 / 3$ for various liquids. The slip lengths all show the same behavior: decreasing wall slip with increasing protrusion angle $\vartheta$. Additionally, the slip lengths are quantitatively similar for all liquids. This is as expected: because of the high flow rate ( $u_{\mathrm{av}}=10^{-1} \mathrm{~m} / \mathrm{s}$ ), in a channel with a height of $H=50 \mu \mathrm{m}$ the shear rates are of the order of $d u / d y=10^{3} 1 / \mathrm{s}$. According to the flow curves given in Fig. 1, the system is thus operated in the upper Newtonian regime and the viscosity of the xanthan gum solutions approaches the value for infinite shear. These results indicate that for aqueous xanthan gum solutions depletioninduced wall slip is either absent or negligible compared to slip arising from superhydrophobicity.
[1] C. L. M. H. Navier, Mem. Acad. Sci. Int. Fr. 6, 389 (1823).

[2] C. Neto, D. R. Evans, E. Bonaccurso, H.-J. Butt, and V. S. J. Craig, Rep. Prog. Phys. 68, 2859 (2005).

[3] J. P. Rothstein, Annu. Rev. Fluid Mech. 42, 89 (2010).

[4] T. Lee, E. Charrault, and C. Neto, Adv. Colloid Interfac. 210, 21 (2014).

[5] R. S. Voronov, D. V. Papavassiliou, and L. L. Lee, Ind. Eng. Chem. Res. 47, 2455 (2008).

[6] Y. Xue, P. Lv, H. Lin, and H. Duan, Appl. Mech. Rev. 68, 030803 (2016).

[7] Y. Cohen and A. B. Metzner, J. Rheol. 29, 67 (1985).

[8] W. Schowalter, J. Non-Newt. Fluid Mech. 29, 25 (1988).

[9] H. A. Barnes, J. Non-Newt. Fluid Mech. 56, 221 (1995).

[10] T. Sochi, Polym. Rev. 51, 309 (2011).

[11] S. G. Hatzikiriakos, Soft Matter 11, 7851 (2015).
[12] F. Brochard and P. G. de Gennes, Langmuir 8, 3033 (1992).

[13] K. B. Migler, H. Hervet, and L. Leger, Phys. Rev. Lett. 70, 287 (1993).

[14] A. L. Yarin, J. Rheol. 42, 1491 (1998).

[15] H. C. Lau and W. R. Schowalter, J. Rheol. 30, 193 (1986).

[16] S. Richardson, J. Fluid Mech. 59, 707 (1973).

[17] Y. Zhu and S. Granick, Phys. Rev. Lett. 88, 106102 (2002).

[18] S. Granick, Y. Zhu, and H. Lee, Nat. Mater. 2, 221 (2003).

[19] J. Sanchez-Reyes and L. A. Archer, Langmuir 19, 3304 (2003).

[20] A. Y. Malkin, Polym. Sci. Ser. A 51, 80 (2009).

[21] R. Tuinier and T. Taniguchi, J. Phys. Condens. Matter 17, L9 (2005).

[22] R. Tuinier, J. K. G. Dhont, T. Taniguchi, and T.-H. Fan, AIP Conf. Proc. 982, 326 (2008). 
[23] F. García-Ochoa, V. E. Santos, J. A. Casas, and E. Gómez, Biotechn. Adv. 18, 549 (2000).

[24] J. de Vicente, J. R. Stokes, and H. A. Spikes, Tribol. Int. 38, 515 (2005).

[25] L. De Vargas and O. Manero, Polym. Eng. Sci. 29, 1232 (1989).

[26] R. Baeza, A. M. R. Pilosof, C. C. Sanchez, and J. M. Rodríguez Patino, AIChE J. 52, 2627 (2006).

[27] D. Ausserré, H. Hervet, and F. Rondelez, Macromolecules 19 85 (1986).

[28] H. Müller-Mohnssen, J. Rheol. 34, 223 (1990).

[29] A. Omari, J. Rheol. 33, 1 (1989).

[30] E. Duering and Y. Rabin, Macromolecules 23, 2232 (1990).

[31] D. Ausserré, J. Edwards, J. Lecourtier, H. Hervet, and F. Rondelez, Europhys. Lett. 14, 33 (1991).

[32] G. Chauveteau, J. Rheol. 26, 111 (1982).

[33] A. M. Jamieson, J. G. Southwick, and J. Blackwell, J. Polym. Sci., A-2, Polym. Phys. 20, 1513 (1982).

[34] M. Milas, M. Rinaudo, and B. Tinland, Polym. Bull. 14, 157 (1985).

[35] G. Cuvelier and B. Launay, Carbohyd. Polym. 6, 321 (1986).

[36] M. Milas, M. Rinaudo, M. Knipper, and J. L. Schuppiser, Macromolecules 23, 2506 (1990).

[37] A. Gamini and M. Mandel, Biopolymers 34, 783 (1994).

[38] S. K. Gulrez, S. Al-Assaf, Y. Fang, G. O. Phillips, and A. P. Gunning, Carbohyd. Polym. 90, 1235 (2012).

[39] J. R. Philip, Z. angew. Math. Phy. 23, 353 (1972).

[40] E. Lauga and H. A. Stone, J. Fluid Mech. 489, 55 (2003).

[41] C. Ybert, C. Barentin, C. Cottin-Bizonne, P. Joseph, and L. Bocquet, Phys. Fluids 19, 123601 (2007).

[42] C. J. Pipe, T. S. Majmudar, and G. H. McKinley, Rheol. Acta 47, 621 (2008).

[43] S. J. Gibbs, D. Xing, T. A. Carpenter, and L. D. Hall, J. Rheol. 38, 1757 (1994).

[44] S. J. Gibbs, J. Rheol. 40, 425 (1996).

[45] C. J. Rofe, J. Rheol. 40, 1115 (1996).

[46] J. Davies, D. Maynes, B. W. Webb, and B. Woolford, Phys. Fluids 18, 087110 (2006).

[47] D. Maynes, K. Jeffs, B. Woolford, and B. W. Webb, Phys. Fluids 19, 093603 (2007).

[48] E. S. Asmolov, S. Schmieschek, J. Harting, and O. I. Vinogradova, Phys. Rev. E 87, 023005 (2013).
[49] C. Schönecker and S. Hardt, J. Fluid Mech. 717, 376 (2013).

[50] C. J. Teo and B. C. Khoo, Microfluid. Nanofluid. 17, 891 (2014).

[51] G. G. Pereira, J. Non-Newt. Fluid Mech. 157, 197 (2009).

[52] S. Dhondi, G. G. Pereira, and S. C. Hendy, Phys. Rev. E 80, 036309 (2009).

[53] A.-L. Vayssade, C. Lee, E. Terriac, F. Monti, M. Cloitre, and P. Tabeling, Phys. Rev. E 89, 052309 (2014).

[54] D. Broboana, N. O. Tanase, and C. Balan, J. Non-Newt. Fluid Mech. 222, 151 (2015).

[55] P. Nghe, E. Terriac, M. Schneider, Z. Li, M. Cloitre, B. Abecassis, and P. Tabeling, Lab Chip 11, 788 (2011).

[56] F. J. Galindo-Rosales, M. A. Alves, and M. S. N. Oliveira, Microfluid. Nanofluid. 14, 1 (2013).

[57] G. D’Avino, F. Greco, and P. L. Maffettone, Annu. Rev. Fluid Mech. 49, 341 (2017).

[58] F. J. H. Gijsen, E. Allanic, F. N. van de Vosse, and J. D. Janssen, J. Biomech. 32, 705 (1999).

[59] G. B. Thurston and N. M. Henderson, Biorheology 43, 729 (2006).

[60] P. Yager, T. Edwards, E. Fu, K. Helton, K. Nelson, M. R. Tam, and B. H. Weigl, Nature 442, 412 (2006).

[61] M. Karle, S. K. Vashist, R. Zengerle, and F. von Stetten, Anal. Chim. Acta 929, 1 (2016).

[62] H. A. Barnes, J. F. Hutton, and K. Walters, An Introduction to Rheology (Elsevier, Amsterdam, 1989).

[63] P. E. Arratia, G. A. Voth, and J. P. Gollub, Phys. Fluids 17, 053102 (2005)

[64] A. M. J. Davis and E. Lauga, Phys. Fluids 21, 011701 (2009).

[65] E. Karatay, A. S. Haase, C. W. Visser, C. Sun, D. Lohse, P. A. Tsai, and R. G. H. Lammertink, Proc. Natl. Acad. Sci. USA 110, 8422 (2013).

[66] T. F. Assis, E. E. G. Rojas, G. C. Guimarães, M. C. Coelho, A. V. Ramos, B. S. Costa, and J. S. R. Coimbra, Int. J. Thermophys. 31, 513 (2010).

[67] L. M. J. Sprakel, Non-Newtonian Flow on Bubble Mattresses, MSc thesis, University of Twente, 2014.

[68] J. A. Schetz and A. E. Fuhs, Fundamentals of Fluid Mechanics (John Wiley \& Sons, New York, 1999).

[69] R. J. Poole and B. S. Ridley, J. Fluids Eng. 129, 1281 (2007). 